\title{
Prevalence and risk factors of the metabolic syndrome in young adults with childhood-onset hypopituitary growth hormone deficiency
}

Han Hyuk Lim, M.D., Min Jae Kang, M.D. ${ }^{2}$, In Suk Yun, M.D. ${ }^{3}$, Young Ah Lee, M.D. ${ }^{2}$, Choong Ho Shin, M.D. ${ }^{2}$, and Sei Won Yang, M.D. ${ }^{2}$

Department of Pediatrics ${ }^{1}$, School of Medicine, Chungnam National University, Daejeon,

Department of Pediatrics' ${ }^{2}$, College of Medicine, Seoul National University, Seoul,

Department of Pediatrics ${ }^{3}$, Gumdan Top General Hospital, Incheon, Korea

Received: 15 July 2010, Revised: 22 August, 2010

Accepted: 8 September 2010

Corresponding author: Choong Ho Shin, M.D.

Department of Pediatrics, College of Medicine, Seoul National University, 28 Yeongun-dong, Jongno-gu, Seoul 110-769, Korea

Tel: +82.2-2072-3371, Fax: +82.2-743-3455

E-mail: chshinpd@snu.ac.kr

Copyright ( 2010 by The Korean Pediatric Society
Purpose: This study evaluated the prevalence of the metabolic syndrome (MetS) and risk factors for metabolic derangement in young adults with childhood-onset hypopituitary growth hormone deficiency (ACOHGHD).

Methods: Thirty patients with ACOHGHD who were treated with hormone-replacement therapy, aged 18 to 29 years, who visited the Seoul National University Children's Hospital between September 2009 and February 2010 were enrolled. Height, weight, waist circumference, hip circumference, and blood pressure were measured, and the clinical and hormonal features were reviewed retrospectively. We evaluated measures of metabolic derangement in the enrolled patients and in the data of healthy adults aged 20 to 29 years taken from the 2005 Korean National Health and Nutrition Examination Survey (KNHANES) as part of the National Cholesterol Education Program-the Adult Treatment Panel III.

Results: Compared with the KNHANES participants, patients with ACOHGHD had significantly large waist circumference (men and women), high systolic blood pressure (BP) (women) and diastolic BP (men), and high serum triglyceride levels (women). The duration of illness correlated significantly with central obesity $\left(r^{2}=0.546, P=0.003\right)$. The prevalence of MetS was $10 \%$ in patients with ACOHGHD and $2.3 \%$ in KNHANES participants. The prevalence of central obesity and MetS was higher in patients with ACOHGHD than in KNHANES participants $(P<0.001$ and $P=0.042$, respectively).

Conclusion: Abdominal obesity correlated with the duration of illness in patients with ACOHGHD. Waist circumference should be measured in the clinic to prevent MetS, particularly in patients with a long history of ACOHGHD, regardless of age or sex.

Key words: Hypopituitarism, Growth hormone deficiency, Metabolic syndrome, Child

This is an open-access article distributed under the terms of the Creative Commons Attribution Non-Commercial License (http://creativecommons.org/licenses/by$\mathrm{nc} / 3.0 /$ ) which permits unrestricted non-commercial use, distribution, and reproduction in any medium, provided the original work is properly cited. 


\section{Introduction}

Adults with hypopituitarism have shorter life expectancy compared with the general population. Growth hormone deficiency (GHD) may contribute to the 1.5- to fourfold increase in cardiovascular mortality and 2.5- to threefold increase in cerebrovascular mortality observed in these patients ${ }^{1-3)}$. Growth hormone $(\mathrm{GH})$ replacement can improve body composition and cardio- and cerebrovascular risk factors associated with $\mathrm{GHD}^{3,4)}$. In children and adolescents with GHD, the primary role of GH is to promote linear growth. In adults, after achieving their final adult height, GH replacement in adults with persistent GHD prevents metabolic imbalances such as those associated with obesity, osteoporosis, and fatty liver ${ }^{5,6}$. The components of the metabolic syndrome (MetS) are central obesity, high blood pressure (BP), high serum triglyceride (TG) level, low serum high density lipoprotein cholesterol (HDLC), and impaired fasting glucose tolerance ${ }^{7,8)}$. GH replacement in patients with GHD may increase BP and insulin and glucose levels, referred to as the insulin-antagonistic effect ${ }^{9}$, or may decrease central obesity. Johannsson and Bengtsson ${ }^{10)}$ noted that the adult GHD phenotype shares features such as abdominal obesity, dyslipidemia, and insulin resistance with the MetS, which represents a cluster of risk factors for cardiovascular disease and type 2 diabetes ${ }^{7,11}$. A recent report showed that the prevalence of the MetS in adult patients with GHD is unaffected by GH replacement, but that baseline MetS status and obesity are strong predictors of the development of the MetS after GH treatment ${ }^{12)}$. However, little is known about metabolic derangements in adult patients with childhood-onset hypopituitary growth hormone deficiency (ACOHGHD) being treated with pituitary hormone replacement including $\mathrm{GH}$.

The aims of this study were to evaluate the prevalence of the MetS and to analyze the risk factors for metabolic abnormalities in patients with ACOHGHD being treated with hormonereplacement therapy (HRT), and to compare relevant variables between patients with ACOHGHD being treated with HRT and healthy young adults.

\section{Materials and methods}

\section{Patients}

The total subject pool was 109 patients (63 men, 46 women) with ACOHGHD being treated with HRT, who visited Seoul National University Children's Hospital between September 2009 and February 2010. Fifty-six of these patients completed the test for MetS during this time, and 30 patients (15 men, 15 women) who met the following criteria were enrolled. The inclusion criteria were that: 1) the patient had achieved a final height confirmed by epiphyseal fusion on wrist $\mathrm{x}$-ray, 2) the patient was 18 to 29 years of age, 3) the patient was deficient in pituitary hormone and was taking replacement therapy, and 4) ACOHGHD was confirmed by a $\mathrm{GH}$ provocation test and the patient was being treated with an adult dose of GH. The exclusion criteria were that: 1) the patient had not been treated with GH from the diagnosis of pituitary GHD, and 2) the patients had chromosomal anomalies or inborn error of metabolism. We used and based on the control data, 639 adults aged 20 to 29 years (264 males, 375 females) from the 2005 Korean National Health and Nutrition Examination Survey (KNHANES).

\section{Methods}

The patients' medical records were examined retrospectively, and the following variables were recorded: age, sex, underlying diseases, treatment methods for underlying diseases, deficient pituitary functions and hormones, and the use of HRT. We measured height, weight, waist circumference, hip circumference, waist-tohip ratio (WHR), BP, and fasting laboratory values such as the serum concentrations of total cholesterol (TC), TG, HDLC, lowdensity lipoprotein cholesterol (LDLC), fasting blood glucose (FBG), insulin, C-reactive protein (CRP), uric acid, aspartate aminotransferase (AST), alanine aminotransferase (ALT), and total bilirubin. Body mass index (BMI) was calculated as the weight (kg) divided by the height (m) squared and is expressed as $\mathrm{kg} / \mathrm{m}^{2}$. Compliance with HRT was evaluated by measuring the serum level of insulin-like growth factor-1 (IGF-1), free thyroxine, folliclestimulating hormone, luteinizing hormone, and testosterone or estradiol. The various components of the Met $S$ were assessed in both the ACOHGHD patients treated with HRT and in the 2005 KNHANES participants. According to the criteria formulated by the National Cholesterol Education Program - the Adult Treatment Panel III (NCEP-ATP III) - the MetS was diagnosed if three or more of the following symptoms were present: 1) central obesity according to the Asian definition (waist circumference $\geq 90 \mathrm{~cm}$ in men or $\geq 80 \mathrm{~cm}$ in women), 2) elevated TG level ( $\geq 150$ $\mathrm{mg} / \mathrm{dL})$, 3) low HDLC level ( $<40 \mathrm{mg} / \mathrm{dL}$ in men or $<50 \mathrm{mg} / \mathrm{dL}$ in women), 4) elevated $\mathrm{BP}$ (systolic $\mathrm{BP} \geq 130 \mathrm{mmHg}$ and/or diastolic $\mathrm{BP} \geq 85 \mathrm{mmHg}$ ), and 5) increased FBG level ( $\geq 100 \mathrm{mg} / \mathrm{dL}$ using the 2006 cutoff of the International Diabetes Federation (IDF) ${ }^{7,8}$.

We defined the cutoff points between thin (BMI <23), overweight $(23 \leq \mathrm{BMI}<25)$, and obesity (BMI $\geq 25)$ using the proposed Asian Pacific Island population cutoff points according to the World Health Organization and the International Obesity Task Force ${ }^{13}$ 14). Waist circumference was measured to the nearest 
$0.1 \mathrm{~cm}$ at the narrowest point between the lowest rib and the uppermost lateral border of the right iliac crest. BP was measured after the subject had rested for 5 minutes in a sitting position and was recorded as the mean of two measurements. The homeostasis model assessment-insulin resistance (HOMA-IR) index and the quantitative insulin-sensitivity check index (QUICKI) for insulin resistance and sensitivity were calculated using the following equations:

HOMA-IR=[fasting glucose $(\mathrm{mmol} / \mathrm{L}) \times$ fasting insulin $(\mathrm{mg} / \mathrm{dL})] / 22.5$

QUICKI $=1 /\left[\log \left(\mathrm{I}_{0}\right)+\log \left(\mathrm{G}_{0}\right)\right]$, where $\mathrm{I}$ is fasting insulin concentration $(\mu \mathrm{U} / \mathrm{mL})$ and $\mathrm{G}_{0}$ is $\mathrm{FBG}$ concentration $(\mathrm{mg} / \mathrm{dL})$.

\section{Statistics}

The patients' age, sex, and duration of illness are presented as mean and standard deviation. The data for underlying diseases and multiple pituitary hormone deficiencies were analyzed using frequency analysis. The Kruskal-Wallis test was used to analyze the changes in the components and related laboratory results of the MetS in subjects categorized by BMI. Components of the MetS were compared between patients and KNHANES participants using an independent $\mathrm{t}$ test or the Mann-Whitney $U$ test. Spearman's correlational analysis and univariate and multivariate linear regression analyses were used to identify the causative factors for central obesity. The relative risk and 95\% confidence interval were analyzed using Fisher's exact test to compare the prevalence of metabolic abnormalities between patients and KNHANES participants. The data were analyzed using SPSS version 12.0 for Windows (SPSS Inc., Chicago, IL, USA), and a $P$ value $<0.05$ was considered significant.

\section{Results}

\section{Clinical information for patients with $A C O H G H D$}

The mean age was 23.1 years and this did not differ between men and women. The mean age of onset of the underlying disease was 9.6 years. The median of BMI was $24.0 \mathrm{~kg} / \mathrm{m}^{2}$ (range, 18.9-39.8). The mean duration of hypopituitary GHD was 15.7 years, and the mean duration of GH treatment was 10.1 years. The underlying diseases of GHD included a germ cell tumor in 10 patients (33.3\%), craniopharyngioma in seven patients (23.3\%), medulloblastoma in four patients (13.3\%), and idiopathic disease in three patients (10.0\%). Before and after the treatment for underlying diseases, 13 patients (43.4\%) developed all form of pituitary dysfunction, such as GHD, hypothyroidism, hypogonadism, hypocortisolism, and diabetes insipidus; seven patients (23.3\%) showed GHD plus one other pituitary hormone deficiency; and three patients $(10.0 \%)$ had isolated GHD (Table 1).

\section{Correlation between parameters of the MetS and BMI}

Three groups (thin, overweight, and obese) were classified according to BMI. Of the various metabolic parameters tested, including waist circumference, hip circumference, WHR, BP, and levels of TC, TG, HDLC, FBG, fasting insulin, CRP, uric acid, AST, ALT, TB, and IGF-1, only WHR correlated significantly with BMI $(P=0.002)$. The level of uric acid $(P=0.078)$ and the ALT $(P=0.052)$ were not significant, but they were associated with BMI. The number of abnormal components of the MetS according to the NCEP-ATP III criteria showed a significant increase according to

Table 1. The Clinical Characteristics of the Patients ( $N=30)$

\begin{tabular}{lc}
\hline Clinical Manifestations & $\mathrm{N}(\%)$ \\
\hline Age (yr) & $23.1(3.1)^{\star}$ \\
Sex (M/F) & $15 / 15$ \\
BMI (kg/m²), median (range) & $24.0(18.9-39.8)$ \\
Onset age of Underlying Disease (yr) & $9.6(4.5)^{\star}$ \\
Duration of GHD (yr) & $15.7(5.4)^{\star}$ \\
Duration of GH treatment (yr) & $10.1(4.7)^{\star}$ \\
Underlying Causes of GHD & \\
Germ Cell Tumor & $10(33.3)$ \\
Craniopharyngioma & $7(23.3)$ \\
Medulloblastoma & $4(13.3)$ \\
Idiopathic & $3(10.0)$ \\
Others & \\
Multiple Pituitary Hormone Deficiency & $6(20.0)$ \\
GHD & \\
GHD+1 Hormone & $3(10.0)$ \\
GHD+2 Hormones & $7(23.3)$ \\
GHD+3 Hormones & $4(13.3)$ \\
GHD+4 Hormones & $3(10.0)$ \\
\hline
\end{tabular}

${ }^{*}$ Mean (SD)

${ }^{\dagger}$ Others include Langerhan's cell histiocytosis, optic glioma, pilocytic astrocytoma, primitive neuroectodermal tumor, agenesis of septum pallucidum, mycobacterial meningitis.

Abbreviations : GHD, growth hormone deficiency

Table 2. Prevalence of the Metabolic Syndrome according to Body Mass Index

\begin{tabular}{lcccc}
\hline Parameters & $\begin{array}{c}\text { Thin } \\
(\mathrm{N}=10)\end{array}$ & $\begin{array}{c}\text { Overweight } \\
(\mathrm{N}=7)\end{array}$ & $\begin{array}{c}\text { Obese } \\
(\mathrm{N}=13)\end{array}$ & $\begin{array}{c}P_{-} \\
\text {value* }\end{array}$ \\
\hline WHR & $0.81(0.05)^{\dagger}$ & $0.90(0.07)^{\dagger}$ & $0.92(0.07)^{\dagger}$ & 0.002 \\
Uric Acid & $5.00(1.18)^{\dagger}$ & $5.57(1.28)^{\dagger}$ & $6.80(2.47)^{\dagger}$ & 0.078 \\
ALT & $11(2-33)^{\ddagger}$ & $17(9-90)^{\ddagger}$ & $20(9-36)^{\ddagger}$ & 0.052 \\
No. of Metabolic Abnormalities & & & & 0.030 \\
$\quad$ factor (\%) & $6(60.0)$ & $2(28.6)$ & $1(7.7)$ & \\
1 facotr (\%) & $2(20.0)$ & $3(42.9)$ & $5(38.5)$ & \\
2 factors (\%) & $2(20.0)$ & $2(28.6)$ & $4(30.8)$ & \\
3 factors (\%) & $0(0.0)$ & $0(0.0)$ & $3(23.1)$ & \\
\hline
\end{tabular}

${ }^{*}$ P-valuse was calculated by Kruskal-Wallis Test

${ }^{\dagger}$ This result represents mean $\pm S D$

†This result means median (range)

Abbreviations : WHR, waist to hip ratio; ALT, alanine aminotransferase 
Table 3. Comparison of Metabolic Components Between ACOHGHD Patients and 2005 KNHANES Participants

\begin{tabular}{|c|c|c|c|c|c|c|}
\hline & \multicolumn{3}{|c|}{ Male } & \multicolumn{3}{|c|}{ Female } \\
\hline & Patients & KNHANES & $P$-value ${ }^{*}$ & Patients & KNHANES & $P$-value* \\
\hline WC (cm) & $89.1(9.4)$ & $79.7(0.6)$ & 0.018 & $82.0(14.4)$ & $72.0(0.6)$ & 0.017 \\
\hline $\mathrm{SBP}(\mathrm{mmHg})$ & $119.7(11.8)$ & $114.9(0.7)$ & 0.133 & $114.1(10.5)$ & $103.9(0.6)$ & 0.002 \\
\hline $\mathrm{DBP}(\mathrm{mmHg})$ & $66.1(6.7)$ & $76.2(0.7)$ & $<0.001$ & $65.7(10.4)$ & $69.1(0.6)$ & 0.231 \\
\hline $\mathrm{TG}(\mathrm{mg} / \mathrm{dL})$ & $110.9(68.0)$ & $113.9(5.2)$ & $0.031^{\dagger}$ & $132.8(64.6)$ & $83.8(2.6)$ & $<0.001^{\dagger}$ \\
\hline $\mathrm{HDLC}(\mathrm{mg} / \mathrm{dL})$ & $50.9(16.6)$ & $44.5(0.7)$ & $0.251^{\dagger}$ & $47.5(11.7)$ & $49.4(0.6)$ & 0.547 \\
\hline $\mathrm{FBG}(\mathrm{mg} / \mathrm{dL})$ & 80.9 (13.2) & $86.3(0.6)$ & 0.139 & $81.9(9.2)$ & $84.4(0.5)$ & 0.315 \\
\hline
\end{tabular}

*This results were analyzed using independent $t$ test

†This results were analyzed using Shapiro-Wilk test and Mann-Whitney $U$ test

Abbreviations : ACOHGHD, young adults with childhood-onset hypopituitary growth hormone deficiency; KNHANES, Korean National Health and Nutrition Examination Survey; WC, waist circumference; SBP, systolic blood pressure; DBP, diastolic blood pressure; TG, triglyceride; HDLC, high density lipoprotein cholesterol; FBG, fasting blood glucose

Mean (SD)

elevated BMI $(P=0.030)$ (Table 2).

\section{The number of abnormal components of the MetS} according to the NCEP-ATP III criteria

In men, waist circumference was significantly higher in patients with ACOHGHD than in KNHANES participants, and diastolic BP and TG level were significantly lower in patients. In women, waist circumference, systolic BP, and serum TG level were significantly higher in ACOHGHD patients than in KNHANES participants. HDLC and FBG levels did not differ between ACOHGHD patients and KNHANES participants. Diastolic BP in men $(P<0.001)$ and TG level in women $(P<0.001)$ differed the most between ACOHGHD patients and KNHANES participants (Table 3).

\section{Risk factors for central obesity in ACOHGHD patients}

Correlational analysis was used to determine whether waist circumference was related to components of the MetS, related laboratory blood tests for the MetS, age, and the duration of illness. Waist circumference correlated significantly with the levels of uric acid and ALT, the number of MetS components, and the duration of illness. However, after controlling for the mutual relationships between variables including age, only the duration of GHD correlated significantly with waist circumference $\left(\mathrm{r}^{2}=0.546\right.$, $P=0.003$ ) (Fig. 1).

\section{Prevalence of individual metabolic risk factors and the} MetS (NCEP-ATP III criteria and the 2006 IDF cutoff) in ACOHGHD patients and 2005 KNHANES participants

The prevalence of central obesity was higher in ACOHGHD patients than in KNHANES participants $(P<0.001)$. Other components did not differ significantly between groups, but hypertension was associated with ACOHGHD $(P=0.054)$. The

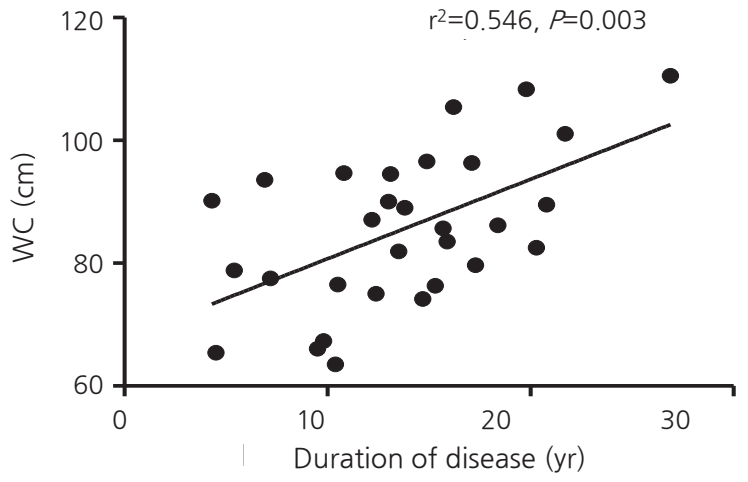

Fig. 1. Correlation between waist circumference and duration of hypopituitarism. Multivariate linear analysis showed that the severity of central obesity correlated positively with the duration of hypopituitary growth hormone deficiency.

Table 4. Prevalence of Metabolic Abnormalities (NCEP-ATP III Criteria) in ACOHGHD Patients and 2005 KNHANES Participants

\begin{tabular}{lrccr}
\hline & $\begin{array}{r}\text { Patients } \\
\mathrm{N}=30(\%)\end{array}$ & $\begin{array}{c}\text { KNHANES } \\
\mathrm{N}=639(\%)\end{array}$ & $\begin{array}{c}\text { Relative Risk } \\
(95 \% \mathrm{Cl})\end{array}$ & $\begin{array}{c}P \text { - } \\
\text { value* }\end{array}$ \\
\hline Abdominal Obesity & $15(50.0)$ & $22(3.4)$ & $14.52(8.42-25.04)$ & $<0.001$ \\
Hypertension & $6(20.0)$ & $57(8.9)$ & $2.24(1.05-4.78)$ & 0.054 \\
Hypertriglyceridemia & $6(20.0)$ & $72(11.3)$ & $1.78(0.84-3.75)$ & 0.147 \\
Low HDL-C & $12(40.0)$ & $230(36.0)$ & $1.11(0.71-1.74)$ & 0.699 \\
Hyperglycemia & $2(6.7)$ & $12(1.9)$ & $3.55(0.83-15.10)$ & 0.127 \\
Metabolic Syndrome & $3(10.0)$ & $15(2.3)$ & $4.26(1.30-13.92)$ & 0.042
\end{tabular}

*This results were analyzed using Fisher's exact test

Abbreviations : ACOHGHD, young adults with childhood-onset hypopituitary growth hormone deficiency; KNHANES, Korean National Health and Nutrition Examination Survey; NCEP-ATP III, National Education Cholesterol Program-Adult Treatment Panel III, HDL, high density lipoprotein; Cl, confidence interval

prevalence of the MetS was significantly higher in ACOHGHD patients than in KNHANES participants (10\% vs $2.3 \%$, respectively, $P=0.042$ ) (Table 4).

\section{Discussion}

This study showed that the prevalence of the MetS was higher 
in ACOHGHD patients treated with an appropriate dose of HRT including GH than in the age-matched general population (10\% vs $2.3 \%$, respectively, $P=0.042$ ). The prevalence of central obesity was significantly higher in the patients than in the general population $(P<0.001)$. Multivariate linear analysis showed a significant correlation between central obesity and the duration of hypopituitarism $\left(\mathrm{r}^{2}=0.546, P=0.003\right)$.

Obesity is a cardinal feature of the MetS. Insulin resistance can be caused by central obesity and can lead to metabolic complications ${ }^{7}$. Previous reports show that in the general population, the prevalence of the MetS increases with age in parallel with age-related increases in the prevalence of obesity and, in particular, central obesity ${ }^{15-17)}$. The HOMA-IR and QUICKI are methods to quantify insulin resistance and sensitivity, respectively ${ }^{18)}$. In this study, central obesity was the most prominent feature that differed between the ACOHGHD patients treated with HRT and the KNHANES participants. However, HOMA-IR, QUICKI, and HDLC and FBG levels did not differ significantly between groups. In the ACOHGHD patients, the severity of central obesity correlated positively with the duration of hypopituitary GHD but not with age. These results suggest the existence of an additional, unidentified cause of the MetS in ACOHGHD patients. Attanasio et $\mathrm{al}^{12)}$ suggested that patients exhibit metabolic abnormalities caused by GHD and possibly inadequate replacement therapy in addition to preexisting, GHD-independent conditions, notably obesity and its consequences. They suggested that it is difficult to establish to what extent the MetS in an individual patient is caused by GHD alone or by preexisting obesity or both. Two recent studies reported no effect of GH replacement on the prevalence of MetS defined by NCEPATP III criteria in GHD patients, ${ }^{190}$. In longitudinal populationbased cohort studies, the persistence of the Met $S$ was found to be variable and to be influenced by factors such as aging, new treatment interventions, and intraindividual variability of laboratory measurements ${ }^{211}$. However, a significant reduction in central obesity, as shown by reduced waist circumference, may reflect the effect of GH treatment ${ }^{22)}$. In patients with hypopituitarism, HRT cannot provide perfect physiological hormone regulation or balance. The difference (mean interval of 5.6 years in our study) between the duration of illness and the duration of GH treatment may influence adverse metabolic consequences such as metabolic syndrome. The MetS may be influenced by the underlying disease itself, drugs such as anticancer agents, antibiotics, immune modulators, radiation therapy, related hypothalamic conditions, and multiple pituitary hormone deficiencies. We hypothesized that the central obesity in ACOHGHD patients with HRT developed because of accumulation of the effects mentioned above and that there would be minimal differences in relation to illness duration. However, in this study, waist circumference or the MetS did not correlate significantly with the underlying diseases, treatment, duration of GH use, or the extent of pituitary dysfunction, although we did not study preexisting obesity.

In the general population, the age-adjusted prevalence of the MetS differs somewhat between women and men, but the direction of that difference is inconsistent between cohorts, ${ }^{7,23}$, ${ }^{24)}$. In contrast to the general population, the women in the present GHD cohort had a higher relative risk for the MetS. Such higher risk in women has been reported in adult hypopituitary GHD patients. A recent study of 750 adult Swedish GHD patients found an increased prevalence of type 2 diabetes mellitus in women compared with men, suggesting that aspects related specifically to GHD or hypopituitarism contribute to this difference ${ }^{9,12,25)}$. The 2005 KNHANES data showed that the prevalence of the Met $S$ in people in their 20 s was $4.7 \%$ of men and $1.2 \%$ of women (The results did not described in Table 3). In our study, systolic $\mathrm{BP}$, diastolic BP, TG level, and the prevalence of the MetS differed between men and women (Table 3). The prevalence of the MetS in the GH-treated ACOHGHD patients aged 18-29 years was $6.7 \%$ in men and $13.3 \%$ in women (The results did not described in Table 3). The higher prevalence of the MetS in women with ACOHGHD might be associated with higher TG levels in these patients than in the KNHANES participants $(P<0.001)$ or in men with ACOHGHD. However, it is unclear whether the higher TG level was an effect of GH treatment or why TG level was higher in women than in men in our study.

A few reports found that the adult-onset form GHD is more likely to be associated with the MetS than is childhood-onset $\mathrm{GHD}^{26-}$ ${ }^{28)}$. These reports suggested that one major reason for this may be the difference in the age ranges of patients in the two onset groups because of the developmental nature of their condition. Childhoodonset GHD patients are exposed to the consequences of GHD or hypopituitarism from childhood and may accumulate metabolic risks. However, the childhood-onset GHD patients' thresholds for abnormaities of the individual MetS components may not be similar to normal adult populations. In this respect, these reports noted that there are limited and inconsistent data on the morbidity and mortality of adult patients with childhood-onset GHD.

We found a significant relationship between waist circumference and the duration of illness, suggesting that they may be important factors when assessing the risk of the MetS in ACOHGHD patients with HRT. The current primary goal in the treatment of hypopituitary GHD patients with HRT is to maintain the normal range of pituitary hormone levels. Our data suggest that the 
patient's waist circumference, an indicator of central obesity, should also be checked as part of the strategy in the prevention of and early intervention for the MetS and related complications, especially in patients with a long history of GHD or hypopituitary illness.

Our study has some limitations. It used a retrospective crosssectional design with a small number of young patients. We did not correct for other possible contributors to the MetS, such as nutrition, physical inactivity, alcohol intake, smoking, socioeconomic status, family history. Long-term follow-up studies of larger groups are needed to understand the changes in metabolic derangement after childhood in these patients.

\section{References}

1) Bülow B, Hagmar L, Mikoczy Z, Nordström CH, Erfurth EM. Increased cerebrovascular mortality in patients with hypopituitarism. Clin Endocrinol (Oxf) 1997;46:75-81.

2) Verhelst J, Abs R. Long-term growth hormone replacement therapy in hypopituitary adults. Drugs 2002;62:2399-412.

3) Erfurth EM, Hagmar L. Cerebrovascular disease in patients with pituitary tumors. Trends Endocrinol Metab 2005;16:334-42.

4) Hoybye C, Jönsson P, Monson JP, Kołtowska-Häggström M, Hana $\mathrm{V}$, Geffner M, et al. Impact of the primary aetiology upon the clinical outcome of adults with childhood-onset GH deficiency. Eur J Endocrinol 2007;157:589-96.

5) Fukuda I, Hizuka N, Yasumoto K, Morita J, Kurimoto M, Takano K. Metabolic co-morbidities revealed in patients with childhood-onset adult GH deficiency after cessation of GH replacement therapy for short stature. Endocr J 2008;55:977-84.

6) Molitch ME, Clemmons DR, Malozowski S, Merriam GR, Shalet SM, Vance ML, et al. Evaluation and treatment of adult growth hormone deficiency: an endocrine society clinical practice guideline. J Clin Endocrinol Metab 2006;91:1621-34.

7) Cornier MA, Dabelea D, Hernandez TL, Lindstrom RC, Steig AJ, Stob NR, et al. The metabolic syndrome. Endocr Rev 2008;29:777-822.

8) Alberti KG, Zimmet P, Shaw J; IDF Epidemiology Task Force Consensus Group. The metabolic syndrome-a new worldwide definition. Lancet 2005;366:1059-62.

9) Holmer H, Svensson J, Rylander L, Johannsson G, Rosén T, Bengtsson BA, et al. Nonfatal stroke, cardiac disease, and diabetes mellitus in hypopituitary patients on hormone replacement including growth hormone. J Clin Endocrinol Metab 2007;92:3560-7.

10) Johannsson G, Bengtsson BA. Growth hormone and the metabolic syndrome. J Endocrinol Invest 1999;22:41-6.

11) Ford ES. Risks for all-cause mortality, cardiovascular disease, and diabetes associated with the metabolic syndrome: a summary of the evidence. Diabetes Care 2005;28:1769-78.

12) Attanasio AF, Mo D, Erfurth EM, Tan M, Ho KY, Kleinberg D, et al. Prevalence of metabolic syndrome in adult hypopituitary growth hormone (GH)-deficient patients before and after $\mathrm{GH}$ replacement. J Clin Endocrinol Metab 2010;95:74-81.

13) WHO Expert Consultation. Appropriate body-mass index for Asian populations and its implications for policy and intervention strategies.
Lancet 2004;363:157-63.

14) Gallagher D. Overweight and obesity BMI cut-offs and their relationship to metabolic disorders in Koreans/Asians. Obes Res 2004;12:440-1.

15) Park YW, Zhu S, Palaniappan L, Heshka S, Carnethon MR, Heymsfield $\mathrm{SB}$. The metabolic syndrome: prevalence and associated risk factor findings in the US population from the Third National Health and Nutrition Examination Survey, 1988-1994. Arch Intern Med 2003;163:427-36.

16) Flegal KM, Carroll MD, Kuczmarski RJ, Johnson CL. Overweight and obesity in the United States: prevalence and trends,1960-1994. Int J Obes Relat Metab Disord 1998;22:39-47.

17) Mokdad AH, Bowman BA, Ford ES, Vinicor F, Marks JS, Koplan JP. The continuing epidemics of obesity and diabetes in the United States. JAMA 2001;286:1195-200.

18) Chen H, Sullivan G, Yue LQ, Katz A, Quon MJ. QUICKI is a useful index of insulin sensitivity in subjects with hypertension. Am J Physiol Endocrinol Metab 2003;284:E804-12.

19) Maison P, Griffin S, Nicoue-Beglah M, Haddad N, Balkau B, Chanson P. Impact of growth hormone $(\mathrm{GH})$ treatment on cardiovascular risk factors in GH-deficient adults: a meta-analysis of blinded, randomized, placebocontrolled trials. J Clin Endocrinol Metab 2004;89:2192-9.

20) van der Klaauw AA, Biermasz NR, Feskens EJ, Bos MB, Smit JW, Roelfsema F, et al. The prevalence of the metabolic syndrome is increased in patients with GH deficiency, irrespective of long term substitution with recombinant human GH. Eur J Endocrinol 2007;156:455-62.

21) Balkau B, Vernay M, Mhamdi L, Novak M, Arondel D, Vol S, et al. The incidence and persistence of the NCEP (National Cholesterol Education Program) metabolic syndrome. The French D.E.S.I.R. study. Diabetes Metab 2003;29:526-32.

22) Carroll PV, Christ ER, Bengtsson BA, Carlsson L, Christiansen JS, Clemmons D, et al.. Growth hormone deficiency in adulthood and the effects of growth hormone replacement: a review. J Clin Endocrinol Metab 1998;83:382-95.

23) Park J, Mendoza JA, O’Neil CE, Hilmers DC, Liu Y, Nicklas TA. A comparison of the prevalence of the metabolic syndrome in the United States (US) and Korea in young adults aged 20 to 39 years. Asia Pac J Clin Nutr 2008;17:471-82.

24) Sesmilo G, Miller K, Hayden D, Klibanski A. Inflammatory cardiovascular risk markers in women with hypopituitarism. J Clin Endocrinol Metab 2001;86:5774-81.

25) Bulow B, Hagmar L, Eskilsson J, Erfurth EM. Hypopituitary females have a high incidence of cardiovascular morbidity and an increased prevalence of cardiovascular risk factors. J Clin Endocrinol Metab 2000;85:574-84.

26) Attanasio AF. Childhood onset adult growth hormone deficiency and hypopituitarism: a black box. In: Webb S, Chanson P, editors. A decade of HypoCCS: the changing face of pituitary disease. Bristol, UK: Bioscentifica Ltd, 2008:61-71.

27) Attanasio AF, Lamberts SW, Matranga AM, Birkett MA, Bates PC, Valk $\mathrm{NK}$, et al. Adult growth hormone (GH)-deficient patients demonstrate heterogeneity between childhood onset and adult onset before and during human GH treatment. J Clin Endocrinol Metab 1997;82:82-8.

28) Besson A, Salemi S, Gallati S, Jenal A, Horn R, Mullis PS, et al. Reduced longevity in untreated patients with isolated growth hormone deficiency. J Clin Endocrinol Metab 2003;88:3664-7. 